\title{
The Coupled Cluster Theory of Quantum Lattice Systems
}

\author{
RAYMOND F. BISHOP and YANG XIAN \\ Department of Mathematics, University of Manchester Institute of Science and Technology (UMIST), \\ P.O. Box 88, Manchester M60 1QD, United Kingdom
}

\begin{abstract}
The coupled cluster method is widely recognized nowadays as providing an ab initio method of great versatility, power, and accuracy for handling in a fully microscopic and systematic way the correlations between particles in quantum many-body systems. The number of successful applications made to date within both chemistry and physics is impressive. In this article we review our recent extensions of the method which now provide a unifying framework for also dealing with strongly interacting infinite quantum lattice systems described by a Hamiltonian. Such systems include both spin-lattice models (such as the anisotropic Heisenberg or $X X Z$ model) exhibiting interesting magnetic properties, and electron lattice models (such as the $t . J$ and Hubbard models), where the spins or fermions are localized on the sites of a regular lattice; as well as lattice gauge theories [such as the Abelian $U(1)$ model of quantum electrodynamics and non-Abelian $S U(n)$ models]. Illustrative resuits are given for both the $X X Z$ spin lattice model and $U(1)$ lattice gauge theory. (c) 1994 John Wiley \& Sons, Inc.
\end{abstract}

\section{Introduction}

The power of the coupled cluster method (CCM) as an ab initio technique in microscopic quantum many-body theory is widely known and well documented in quantum chemistry $[1,2]$, particularly where results of extremely high accuracy are required, such as for the calculation of molecular energy differences of chemical significance or for the calculation of parity violation in atoms. However, the CCM has also been used much more widely in quantum many-body theory and quantum field theory, where it has now become what is probably the most powerful, most universally applicable, and numerically most accurate of all available fully microscopic frameworks for describing in a fully rigorous fashion the quantum correlations between systems of interacting particles. The number of successful applications to date within both chemistry and physics is truly impressive. In most cases the numerical results achieved are either the best or among the best available. A recent review for a quantum chemistry audience of the applications of the CCM outside chemistry has been given by one of the present authors [3]. Bartlett has given a comparable review [1] within chemistry. In addition, there have been many recent reviews of the method itself, including those given in Refs. [3-12].

This widespread success of the CCM applications to both finite and extended systems in continuous space has naturally led to the method being applied in the 
last few years [13-24] to quantum-mechanical systems defined on an extended regular spatial lattice. Examples of such applications include: (i) spin-lattice systems, such as the solid phases of ${ }^{3} \mathrm{He} \mathrm{[13]} \mathrm{and} \mathrm{various} \mathrm{models} \mathrm{of} \mathrm{interest} \mathrm{in} \mathrm{magnetism,}$ e.g., the spin- $\frac{1}{2}$ anisotropic Heisenberg (or $X X Z$ ) model [14] and its extension to higher spins [15], and the spin-1 Heisenberg-biquadratic model [16]; (ii) models of strongly interacting electrons on lattices, such as the Hubbard model [19-21]; and (iii) lattice gauge field theories [22-24].

The recent discovery of high-temperature superconductors has played a large part in the resurgence of interest in spin and electron lattice models. For example, among the various models proposed, the Hubbard model of electrons on a twodimensional (2D) square lattice is widely believed to describe the essential correlations of the active electrons in these ceramic cuprate materials. Furthermore, the antiferromagnetic Heisenberg model of spins on a lattice can be derived from the Hubbard model at half-filling, and is itself believed to describe the electronic properties of the undoped insulating precursors to the high- $T_{c}$ materials.

We also note that even quantum-mechanical spin-lattice systems with very simple Hamiltonians are often endowed with very complicated physical properties and intricate phase diagrams. Their behavior is often quite unlike that of their classical counterparts. An obvious example is the one-dimensional (1D) Heisenberg model, which is fully integrable by the Bethe ansatz technique [25] in the spin- $\frac{1}{2}$ case, from which it is known to have a gapless excitation spectrum, but which is conjectured [26] to have a nonzero gap in the nonintegrable spin-1 case. More generally, such quantal spin systems often exhibit a rich variety of orderings and phases as functions of the combinations of interactions (e.g., nearest-neighbor plus nextnearest-neighbor), the lattice structure, the spin of the particles, the number of spatial dimensions, and such other external variables as the applied magnetic field. A single system can thus display one or more of: phases with Ising-like long-range order, dimerized or trimerized phases, valence-bond solid structure, and much other exotic and often only poorly understood structure. In the light of both this richness of behavior and the existence of various exact solutions for several models with certain values of their parameters, spin-lattice models provide a particular challenge for quantum many-body theory to provide an accurate and detailed microscopic description of their manifold properties.

A comparable challenge is provided by lattice gauge field theory, which was originally introduced to provide a cure for the chronic divergences inherent in comparable field theories in a spatial continuum. Lately, lattice gauge models have become of interest in their own right, partly because they provide an especially severe test for theoretical techniques due to their intrinsically nonperturbative character and the limitations imposed by the requirement to impose manifest gaugeinvariance at all stages. However, lattice gauge models share many of the features and attributes of spin-lattice models. It has been our intention to exploit this similarity and to develop the CCM as a unifying framework for treating all infinitelattice Hamiltonians. In particular, we have shown how various CCM lattice approximation schemes developed for the spin models can be simply modified for use with both Abelian and non-Abelian lattice gauge theories. 
The remainder of this article is organized as follows. In the next section we describe the basic elements of the CCM which are needed for the analysis of a general many-body system. In the third section we consider in some detail the $X X Z$ model Hamiltonian as an archetypal spin-lattice problem to which the CCM can be applied. Particular attention is paid to the development of suitable approximation schemes. We also show that these lead to results of very high accuracy at relatively low orders of implementation. In the subsequent section we demonstrate how analogues of these schemes may be applied with comparable success to lattice gauge theories, where we choose the $U(1)$ model as a concrete example. Our findings are summarized in the final section, where we also indicate possible further extensions of the CCM and consider its potential for even wider applications.

\section{Basic Elements of the CCM Formalism}

In order to describe quantitatively the multiparticle correlations in a quantum many-body system one always needs a model (or reference) state $|\Phi\rangle$ against which to compare them. In the CCM the only requirement on $|\Phi\rangle$ is that it forms a cyclic vector with respect to which we may define two subalgebras, namely that of the multiconfigurational creation operators $\{C\}\}$ and that of their Hermitian-conjugate destruction counterparts $\left\{C_{J}\right\}$. Very importantly, both subalgebras are required to be Abelian, i.e., $\left.[C\}, C_{J^{\prime}}^{\dagger}\right]=0=\left[C_{J}, C_{J^{\prime}}\right]$, where each set-index $J$ labels a general multiparticle cluster configuration with respect to $|\Phi\rangle$, which itself thus plays the role of vacuum (or reference) state. We thus require that the operators $\left\{C_{J}^{\dagger}\right\}$ and $\left\{C_{J}\right\}$ are complete, in the sense that arbitrary ket and bra states within the appropriate many-body Hilbert space $\mathscr{H}$ may be decomposed as the respective linear combinations,

$$
|\Psi\rangle=\sum_{J} f_{J} C \zeta|\Phi\rangle ;\langle\tilde{\Psi}|=\sum_{J} \tilde{f}_{J}\langle\Phi| C_{J}
$$

This is clearly achievable if the identity operator $I$ within $\mathscr{H}$ has the resolution,

$$
I=\sum_{J} C_{J}^{\dagger}|\Phi\rangle\langle\Phi| C_{J}
$$

For practical purposes it is also convenient, but not essential, if the operators form an orthonormal set, in the following sense,

$$
\left\langle\Phi\left|C_{J} C_{J^{\prime}}^{\dagger}\right| \Phi\right\rangle=\delta\left(J, J^{\prime}\right),
$$

where $\delta\left(J, J^{\prime}\right)$ is some (suitably defined) delta function between the set indices $\{J\}$.

As an example we consider the familiar application of the CCM to an $\mathrm{N}$-electron molecule. Here $|\Phi\rangle$ is chosen as an $N$-electron Slater determinant formed from the lowest $N$ levels of an (orthonormal) set of suitably defined molecular (spin-) orbitals. The set-indices in the fixed- $N$ Hilbert space, $J \rightarrow\left\{p_{1}, \cdots, p_{m} ; \alpha_{i}, \cdots\right.$, $\left.\alpha_{m}\right\}$, now represent an $m$-particle/ $m$-hole cluster configuration, where the singleparticle indices $\left\{\alpha_{i}\right\}$ label the single-hole states that can be created by removing 
an electron from $|\Phi\rangle$, and the single-particle indices $\left\{p_{i}\right\}$ label the remaining unoccupied or particle states.

The CCM is now characterized by writing the exact ground ket state $\left|\Psi_{0}\right\rangle$ of the many-body Hamiltonian $H$ in terms of an exponentiated cluster correlation operator $S$,

$$
\left|\Psi_{0}\right\rangle=e^{S}|\Phi\rangle ; \quad H\left|\Psi_{0}\right\rangle=E_{0}\left|\Psi_{0}\right\rangle,
$$

which may be decomposed wholly and exactly in terms of creation operators,

$$
S=\sum_{J}{ }^{\prime} \mathcal{S}_{J} C J
$$

where, by definition, the prime on the sum over configurations $\{J\}$ excludes the $J=0$ term corresponding to the identity operator, $C_{0}^{\dagger} \equiv I$. We thus have the intermediate normalization condition, $\left\langle\Phi \mid \Psi_{0}\right\rangle=\langle\Phi \mid \Phi\rangle=1$, in the usual scheme where $\left\langle\Phi\left|C_{J}^{\dagger}\right| \Phi\right\rangle=0, \forall J \neq 0$. The correlation operator of an $N$-body system may itself also be decomposed as a linear superposition of $n$-body partitions, $S=$ $\sum_{n=1}^{N} S_{n}$, where $S_{n}$ describes the configurations formed by exciting $n$ particles from their single-particle states occupied in $|\Phi\rangle$.

The reason for writing the correlation operator in the exponential form of Eqs. (4) and (5), rather than in the linear form of Eq. (1) which is typical of the configuration-interaction $(\mathrm{CI})$ method, lies at the heart of the CCM [3,5,7]. Particularly important features of the exponential form are: (i) its proper counting of independent excitations (e.g., $n$ independent pairs of particles excited from $|\Phi\rangle$ are properly described by the wave function $\left.(1 / n !) S_{2}^{n}|\Phi\rangle\right)$; (ii) its automatic incorporation of the important linked-cluster theorem of Goldstone; and (iii) its incorporation of size-extensivity, so that such extensive variables as the ground-state energy $E_{0}$ scale properly with particle number in the infinite limit $N \rightarrow \infty$, even when $S$ is truncated.

In practice the CCM is implemented by restricting the cluster amplitudes $\left\{\mathcal{S}_{J}\right\}$ to some (finite or infinite) subset of the entire set, within a well-defined hierarchy of such approximations. Solutions for the amplitudes retained are then found by first rewriting the ground-state Schrödinger Eq. (4) in the similarity-transformed form,

$$
\left(\mathrm{e}^{-S} H \mathrm{e}^{S}-E_{0}\right)|\Phi\rangle=0,
$$

which underpins the CCM. By taking the inner products of Eq. (6) with the complete set of states comprising $|\Phi\rangle$ itself and $\left\{C_{J}^{\dagger}|\Phi\rangle ; J \neq 0\right\}$, we find, respectively,

$$
\begin{gathered}
\left\langle\Phi\left|\mathrm{e}^{-S} H \mathrm{e}^{S}\right| \Phi\right\rangle=E_{0}, \\
\left\langle\Phi\left|C_{\mathcal{J}} \mathrm{e}^{-S} H \mathrm{e}^{S}\right| \Phi\right\rangle=0 ; \quad J \neq 0 .
\end{gathered}
$$

The set of Eqs. (8) represents a coupled set of nonlinear equations for the $c$-number coefficients $\left\{\mathfrak{S}_{J}\right\}$, equal in number to the number of retained configurations $\{J\}$. In terms of their solutions, Eq. (7) then gives the corresponding approximant for the ground-state energy, $E_{0}=E_{0}\left[\mathscr{S}_{J}\right]$.

The well-known expansion in terms of nested commutators, 


$$
\mathrm{e}^{-S} H \mathrm{e}^{S}=H+[H, S]+\frac{1}{2 !}[[H, S], S]+\cdots,
$$

together with the fact that each of the individual terms in the expansion of Eq. (5) for the operator $S$ commutes with all others, ensures that every element of $S$ in Eq. (5) is linked directly to the Hamiltonian in each of the terms in Eq. (9). No unlinked (or, equivalently, disconnected) terms or diagrams can arise, and each of the coupled equations ( 8 ) is of linked-cluster type. Furthermore, when each term in the Hamiltonian $H$ in second-quantized form contains only a finite number of single-particle destruction operators defined with respect to $|\Phi\rangle$, as is usually the case, the otherwise infinite series of Eq. (9) will always terminate after a finite number of terms. As a consequence, the left-hand sides of Eqs. (7) and (8) are expressions of finite order in the retained coefficients $\left\{\mathcal{S}_{J}\right\}$, and no further (artificial or approximate) truncation is required for their evaluation. The CCM thus fundamentally differs from its unitarity-transformation counterpart that would arise in a standard variational formulation, in which the bra state $\left\langle\Psi_{0}\right|$ is simply taken as the manifest Hermitian conjugate, $\langle\Phi| \mathrm{e}^{S^{\dagger}}$, of $\left|\Psi_{0}\right\rangle$.

The above feature is very important, and leads naturally to the biorthogonal description of states within the CCM (rather than the more usual orthogonal description), in which the manifest Hermitian conjugacy of corresponding bra and ket states is lost. While the bra states are not needed to calculate the ground-state energy, they are needed to calculate such other ground-state expectation values as order parameters. In what has become known [5] as the normal version of the CCM, the bra ground-state is parametrized in the form,

$$
\begin{gathered}
\left\langle\tilde{\Psi}_{0}\right|=\langle\Phi| \tilde{S} \mathrm{e}^{-S} ; \quad\left\langle\tilde{\Psi}_{0}\right| H=E_{0}\left\langle\tilde{\Psi}_{0}\right|, \\
\tilde{S}=1+\sum_{J} \tilde{\mathcal{S}}_{J} C_{J},
\end{gathered}
$$

with explicit normalization,

$$
\left\langle\tilde{\Psi}_{0} \mid \Psi_{0}\right\rangle=\left\langle\Phi \mid \Psi_{0}\right\rangle=\langle\Phi \mid \Phi\rangle=1 .
$$

Equation (10) readily leads to the equations,

$$
\left\langle\Phi\left|\tilde{S}\left(\mathrm{e}^{-S} H \mathrm{e}^{s}-E_{0}\right) C_{J}^{\dagger}\right| \Phi\right\rangle=0, \quad \forall J,
$$

which, by making use of Eq. (7) and the fact that $\left[S, C_{\zeta}^{\dagger}\right]=0$ for all set-indices $J$, may easily be rewritten as,

$$
\left\langle\Phi\left|\tilde{S} \mathrm{e}^{-S}[H, C \zeta] \mathrm{e}^{S}\right| \Phi\right\rangle=0, \quad \forall J .
$$

Equations (13) and (14) have the form of coupled but linear equations for the brastate coefficients $\left\{\tilde{\mathcal{S}}_{J}\right\}$ once the ket-state coefficients $\left\{\mathcal{S}_{J}\right\}$ are used as known input. Once again, the resulting equations contain only finite powers of $S$ since the nested commutator expansions again terminate as before. It is not difficult to show that Eq. (13) has the formal solution 


$$
\begin{gathered}
\langle\Phi| \tilde{S}=\langle\Phi|-\langle\Phi| \mathrm{e}^{-S} H \mathrm{e}^{S} Q\left(Q \mathrm{e}^{-S} H \mathrm{e}^{S} Q-E_{0}\right)^{-1} Q, \\
Q \equiv I-|\Phi\rangle\langle\Phi|,
\end{gathered}
$$

in terms of which the ground-state expectation value of an arbitrary operator $A$ can be written as

$$
\begin{aligned}
\bar{A} & =\bar{A}\left[\mathscr{S}_{J}\right] \equiv\left\langle\tilde{\Psi}_{0}|A| \Psi_{0}\right\rangle \\
& =\left\langle\Phi\left|\mathrm{e}^{-S} A \mathrm{e}^{S}\right| \Phi\right\rangle-\left\langle\Phi\left|\mathrm{e}^{-S} H \mathrm{e}^{S} Q\left(Q \mathrm{e}^{-S} H \mathrm{e}^{S} Q-E_{0}\right)^{-1} Q \mathrm{e}^{-S} A \mathrm{e}^{S}\right| \Phi\right\rangle .
\end{aligned}
$$

We note that Eq. (8) guarantees that $\bar{H}=E_{0}$, as given by Eq. (7).

Excited states $\left\{\left|\Psi_{\lambda}\right\rangle\right\}$ are described within the CCM [27] by linear excitation operators $\left\{X^{\lambda}\right\}$ which act on the corresponding exact ground-state wave function $\left|\Psi_{0}\right\rangle$,

$$
\left|\Psi_{\lambda}\right\rangle=X^{\lambda}\left|\Psi_{0}\right\rangle=X^{\lambda} \mathrm{e}^{S}|\Phi\rangle ; \quad H\left|\Psi_{\lambda}\right\rangle=E_{\lambda}\left|\Psi_{\lambda}\right\rangle \equiv\left(E_{0}+\varepsilon_{\lambda}\right)\left|\Psi_{\lambda}\right\rangle,
$$

where $X^{\lambda}$ is again decomposed wholly in terms of creation pieces with respect to $|\Phi\rangle$,

$$
X^{\lambda}=\sum_{J}^{\prime} \chi_{\delta}^{\lambda} C_{J}^{\dagger}
$$

Hence the operators $S$ and $X^{\lambda}$ commute. The excited-state Schrödinger Eq. (18) may be combined with its ground-state counterpart of Eq. (4) to give the CCM eigenvalue equation,

$$
\mathrm{e}^{-S}\left[H, X^{\lambda}\right] \mathrm{e}^{S}|\Phi\rangle=\varepsilon_{\lambda} X^{\lambda}|\Phi\rangle,
$$

for the excitation energy, $\varepsilon_{\lambda} \equiv E_{\lambda}-E_{0}$, directly. Equation (20) may be equivalently written as,

$$
\left(Q \mathrm{e}^{-S} H \mathrm{e}^{S} Q-E_{0}\right) Q X^{\lambda}|\Phi\rangle=\varepsilon_{\lambda} Q X^{\lambda}|\Phi\rangle,
$$

from which we see that the excitation energies are given by diagonalizing in the space spanned by the states $\{C \zeta|\Phi\rangle ; J \neq 0\}$ the same matrix $\left(Q \mathrm{e}^{-S} H \mathrm{e}^{S}-E_{0}\right)$ as needs to be formally inverted in Eq. (15) to find $\tilde{S}$. In all practical implementations of the CCM, this space is truncated to some (finite or infinite) subspace spanned by a subset of the states. The operators $S, \hat{S}$, and $X^{\lambda}$ given by Eqs. (5), (11), and (19) are thus approximated by restricting the sum over configurations $\{J\}$ to the appropriate subset.

In the following two sections we show how the above general CCM scheme can be applied to both spin-lattice Hamiltonian systems and Hamiltonian lattice gauge theories.

\section{Applications to Spin Lattices}

We illustrate the application of the СCM to spin-lattice problems by considering the archetypal $X X Z$ or anisotropic Heisenberg model as a specific example. This model comprises $N$ spins, with spin quantum number $s$, on the sites $\{l\}$ of a $d$ - 
dimensional regular lattice, which interact via nearest-neighbor couplings described by the Hamiltonian

$$
H=\frac{1}{2} \sum_{l=1}^{N} \sum_{\rho=1}^{z}\left[\Delta s_{l}^{z} s_{l+\rho}^{z}+\frac{1}{2}\left(s_{l}^{+} s_{l+\rho}^{-}+s_{l}^{\bar{s}} s_{l+\rho}^{+}\right)\right],
$$

where the index $l$ runs over all $N(\rightarrow \infty)$ lattice sites with the usual periodic boundary condition imposed; the index $\rho$ runs over all $z$ nearest-neighbor sites (i.e., $z$ is the coordination number of the lattice); the operators $s_{l}^{z}$ and $s_{l}^{ \pm} \equiv s_{l}^{x} \pm i s_{l}^{y}$ are spin-s operators which obey the usual $S U(2)$ angular momentum commutation relations; and $\Delta$ is the anisotropy parameter, which in the special case $\Delta=1$ gives the isotropic Heisenberg model. For present purposes we consider only bipartite lattices, where the lattice is composed of two equivalent alternating sublattices, denoted as $A$ and $B$. Bipartite lattices thus include the important $1 \mathrm{D}$ chain and the $2 \mathrm{D}$ square lattice. The restriction to bipartite lattices excludes such lattices as the triangular lattice and such associated effects as frustration. We also restrict attention here to the case $\Delta>0$, where for the 1D chain, for example, the system is known to be antiferromagnetic in its ground state.

In order to perform a CCM analysis of this model we must now first choose a model state $|\Phi\rangle$. Although there are many alternative choices [16], we illustrate the approach here with what is possibly the simplest and most obvious choice, namely where $|\Phi\rangle$ is chosen to be the (classical) Néel state $|N\rangle$ in which all spins on the $A$-sublattice have $z$-component $s^{z}=-s$, say, and all those on the $B$-sublattice have $s^{z}=+s$. This state thus has perfect antiferromagnetic long-range order, but is an eigenstate of the quantum Hamiltonian of Eq. (22) only in the Ising model $(\Delta \rightarrow \infty)$ limit. The single-spin creation operators with respect to $|N\rangle$ are clearly the spin-raising operators $\left\{s_{i}^{+}\right\}$for sites $i$ on the "spin-down" $A$-sublattice and the spin-lowering operators $\left\{s_{j}^{-}\right\}$for sites $j$ on the "spin-up" $B$-sublattice. Each of these operators can act a maximum of $2 s$ times on a single site before the state $|N\rangle$ is annihilated.

The $X X Z$ Hamiltonian of Eq. (22) is easily seen to commute with the operator $s_{\text {total }}^{z} \equiv \sum_{l=1}^{N} s_{l}^{z}$. Hence, the antiferromagnetic ground-state $\left|\Psi_{0}\right\rangle$ may, by definition, be sought in the subspace where $s_{\text {total }}^{z}=0$. Equations (4) and (5) thus become, in this case,

$$
\left|\Psi_{0}\right\rangle=\mathrm{e}^{S}|N\rangle ; \quad S=\sum_{m=1}^{N / 2} S_{2 m}
$$

with cluster correlation operators $S_{2 m}$ expressed with a somewhat arbitrary choice of normalization constant as,

$$
S_{2 m}=\frac{(-1)^{m}}{(m !)^{2}} \sum_{i_{1}, i_{2}, \cdots i_{m}} \sum_{j_{1}, j_{2}, \cdots j_{m}} \rho_{i_{1} i_{2}} \cdots i_{m}, j_{1} j_{2} \cdots j_{m} s_{i_{1}}^{+} s_{i_{2}}^{+} \cdots s_{i_{m}}^{+} s_{j_{1}}^{-} s_{j_{2}}^{-} \cdots s_{j_{m}}^{-},
$$

where indices $\left\{i_{l}\right\}$ and $\left\{j_{m}\right\}$ refer to sites on the $A$ - and $B$-sublattices, respectively. The equivalent analogues of Eqs. (10) and (11) are, 


$$
\begin{aligned}
& \left\langle\tilde{\Psi}_{0}\right|=\langle N| \tilde{S} \mathrm{e}^{-S} ; \quad \tilde{S}=1+\sum_{m=1}^{N / 2} \tilde{S}_{2 m},
\end{aligned}
$$

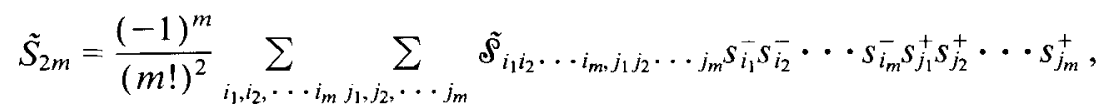

Clearly, for spin- $s$ particles the coefficients $\mathcal{S}_{i_{1}} \cdots i_{m}, j_{1} \cdots j_{m}$ and $\tilde{\mathcal{S}}_{i_{1}} \cdots i_{m}, j_{1} \cdots j_{m}$ vanish if any one of the lattice indices appears more than $2 s$ times.

The ground-state energy per particle may simply be calculated from Eq. (7). We find,

$$
\frac{E_{0}}{N}=-\frac{z s^{2}}{2}\left(\Delta+2 b_{1}\right)
$$

where, as before, $z$ is the lattice coordination number, and $b_{1} \equiv \mathfrak{S}_{i, i+\rho}$ is the nearestneighbor pair correlation coefficient. We note that $b_{1}$ is independent of both the index $i$ (by lattice translational invariance) and the index $\rho$ (by the lattice symmetries under rotations and reflections). We also note that Eq. (27) is exact. The coefficient $b_{1}$ is itself calculated from the coupled sets of equations for the coefficients $\left\{\oint_{i_{1}} \cdots i_{n}, j_{1} \cdots j_{n}\right\}$, which are determined as in Eq. (8), with $C_{J} \rightarrow s_{i_{1}}^{-} \cdots$ $s_{i_{n}}^{-} s_{j_{1}}^{+} \cdots s_{j_{n}}^{+}$. As usual the number of such equations must be truncated in practice by restricting the configurations retained in Eqs. (24) and (26), and then solving Eqs. (8) and (14) without further approximations, keeping in each case the same configurations for these coupled sets of equations, so that we have one equation in each set for each retained ket- or bra-state coefficient. The algebraic form of the sets of Eqs. (8), for example, is that of a coupled set of multinomial equations, each term of which is easily seen from Eq. (9) to be of degree no higher than fourth, by noting the bilinear form of the Hamiltonian of Eq. (22) and the specific $S U(2)$ commutation relations. Their explicit evaluation is straightforward, although very tedious and time-consuming if done by hand if more than a few configuration coefficients are retained. Nevertheless, the algebraic reduction is very amenable to automation using computer-algebraic techniques, and many of the actual results given below have been found this way.

All that remains at this point, in order to perform a CCM calculation, is to consider appropriate truncation hierarchies. These cannot ever be uniquely specified, and must always be motivated on physical grounds for the problem at hand. We discuss here two such schemes. From previous applications of the CCM to systems defined in a spatial continuum, the most well-known truncation hierarchy is the so-called SUB $n$ scheme defined by truncating the summations in Eqs. (23) and (25) so that one retains only up to $n$-body correlation coefficients,

$$
S \rightarrow S_{\mathrm{SUB} n} \equiv \sum_{k=1}^{n} S_{k} ; \quad \tilde{S} \rightarrow \tilde{S}_{\mathrm{SUB} n} \equiv 1+\sum_{k=1}^{n} \tilde{S}_{k}
$$

For example, the CCM coupled equations for the two-spin-flip ket-state coefficients $\mathcal{f}_{i, j}$ are now found from the relation, 


$$
\left\langle N\left|s_{i}^{-} s_{j}^{+} \mathrm{e}^{-S_{2}} \mathrm{He}^{S_{2}}\right| N\right\rangle=0 .
$$

Straightforward evaluation of Eq. (29) yields the coupled equations,

$$
\sum_{\rho}\left[\left(1+2 \Delta b_{1}+2 b_{1}^{2}\right) \delta_{\rho, r}-4 s\left(\Delta+2 b_{1}\right) b_{r}+4 s^{2} \sum_{r^{\prime}} b_{r^{\prime}} b_{r-r^{\prime}+\rho}\right]=0,
$$

where $b_{r} \equiv \mathfrak{S}_{i, j}$ with $r \equiv j-i$, and we have taken advantage of the translational invariance of the lattice. We note that in $1 \mathrm{D} r$ is simply a (positive or negative) odd integer, whereas in $2 \mathrm{D} r$ is a lattice vector connecting sites on opposite sublattices. Rotational and reflectional symmetry imposes extra relations among the coefficients $\left\{b_{r}\right\}$ : for example, in $1 \mathrm{D}, b_{-r}=b_{r}$. The bra-state coefficients $\tilde{b}_{r} \equiv \tilde{\mathfrak{S}}_{i, j}$ are defined, and equivalent relations derived, similarly.

The full set of nonlinear SUB2 Eqs. (30) can be solved analytically by a sublattice Fourier transform method [14]. For the 1D chain, for example, the solution is

$$
\begin{gathered}
b_{r}=K \int_{0}^{\pi} \frac{d q}{\pi} \frac{\cos r q}{\cos q}\left(1-\sqrt{1-k^{2} \cos ^{2} q}\right), \\
K \equiv \frac{1}{2 s}\left(\Delta+2 b_{1}\right), \quad k^{2} \equiv \frac{1+2 \Delta b_{1}+2 b_{1}^{2}}{\left(\Delta+2 b_{1}\right)^{2}} .
\end{gathered}
$$

The coefficient $b_{1}$ is thus determined self-consistently by putting $r=1$ in Eq. (31), from which the ground-state energy may be immediately found from Eq. (27). Results for the 1D spin- $\frac{1}{2}$ chain and the 2D spin- $\frac{1}{2}$ square lattice are given in Tables I and II, respectively. Very interestingly, the exact SUB2 solution of Eq. (31) predicts a phase transition at the terminating point in Eq. (31) for which $k \rightarrow k_{c}=1$, and beyond which the equation has no real solution. This terminating condition determines a critical anisotropy $\Delta_{c}$, such that no real SUB2 solution exists for $\Delta<\Delta_{c}$. Numerical solutions yield the values $\Delta_{c} \approx 0.3728,0.7985$, and 0.8872 for the spin$\frac{1}{2}$ models on the $1 \mathrm{D}$ chain, $2 \mathrm{D}$ square lattice, and $3 \mathrm{D}$ cubic lattice, respectively.

TABLE I. Ground-state energy per spin as a function of $\Delta$ for the 1D spin- $\frac{1}{2} X X Z$ model. Shown are the results of the full SUB2 and various LSUB $n$ schemes, defined in the text, together with the exact results for comparison.

\begin{tabular}{lcccccc}
\hline \hline & \multicolumn{7}{c}{$\Delta$} \\
\cline { 2 - 7 } Method & 0.0 & 0.5 & 1.0 & 1.5 & 2.0 & 5.0 \\
\hline SUB2 & - & -0.3506 & -0.4186 & -0.5075 & -0.6079 & -1.2986 \\
LSUB2 & -0.2887 & -0.3412 & -0.4167 & -0.5069 & -0.6076 & -1.2986 \\
LSUB4 & -0.3193 & -0.3692 & -0.4363 & -0.5195 & -0.6155 & -1.2995 \\
LSUB6 & -0.3198 & -0.3730 & -0.4400 & -0.5218 & -0.6167 & -1.2995 \\
LSUB8 & -0.3196 & -0.3741 & -0.4414 & -0.5226 & -0.6170 & -1.2995 \\
LSUB10 & -0.3194 & -0.3745 & -0.4420 & -0.5230 & -0.6171 & -1.2995 \\
Exact & -0.3183 & -0.3750 & -0.4432 & -0.5234 & -0.6172 & -1.2995 \\
\hline \hline
\end{tabular}


TABLE II. Ground-state energy per spin for the $2 \mathrm{D}$ spin- $\frac{1}{2}$ Heisenberg model $(\Delta=1)$ on a square lattice. Shown are the results of the full SUB2 and various $L S U B(w, d)$ schemes, defined in the text, together with the results of spin-wave theory (SWT) of Ref. [29], and from the Green's function Monte Carlo (GFMC) calculations of Ref. [28] for comparison.

\begin{tabular}{lccccc}
\hline \hline SUB2 & LSUB(6,6) & LSUB $(8,8)$ & LSUB(10,10) & SWT & GFMC \\
\hline-0.6508 & -0.6483 & -0.6531 & -0.6644 & -0.6580 & -0.6692 \\
\hline
\end{tabular}

We have shown elsewhere $[14,15]$, by the further calculation of the spin-spin correlation functions, the staggered magnetization, and the low-lying excitations, that the above critical point really corresponds to the known Ising-Heisenberg phase transition, which is known to occur at $\Delta=1$ in the $1 \mathrm{D}$ spin- $\frac{1}{2}$ chain. (We also note that when more of the higher-order multi-spin correlations neglected in SUB2 approximation are included, the value of $\Delta_{c}$ moves closer to this exact value [14], as expected.) For example, we have shown by calculating from Eqs. (18) and (19) the spin-wave-like excitations on top of the SUB2 ground state, that the lowlying excitation spectrum has a finite gap for $\Delta>\Delta_{c}$ and that this gap exactly vanishes as $\Delta \rightarrow \Delta_{c}$ for lattices of all dimensions. Furthermore, the staggered magnetization $M^{z}$, defined for a spin on the site $j$ on the ("up") $B$-sublattice as,

$$
M^{z} \equiv \frac{1}{S}\left\langle s_{j}^{z}\right\rangle=\frac{1}{s}\left\langle\Phi\left|\tilde{S}^{-S} S_{j}^{z} \mathrm{e}^{S}\right| \Phi\right\rangle,
$$

can easily be evaluated in the SUB2 approximation as,

$$
M_{\mathrm{SUB} 2}^{z}=1-4 s \sum_{r} \tilde{b}_{r} b_{r} .
$$

The exact solution of Eq. (31) for $\left\{b_{r}\right\}$ and its counterpart for $\left\{\tilde{b}_{r}\right\}$ yield that $M_{\mathrm{SUB} 2}^{z} \rightarrow 0$ as $\Delta \rightarrow \Delta_{c}$ in the $1 \mathrm{D}$ case, in agreement with the exact result [25] at $\Delta=1$, but that $M_{\mathrm{SUB} 2}^{z}$ remains nonzero as $\Delta \rightarrow \Delta_{c}$ in 2D and 3D. For example, for the important $2 \mathrm{D}$ spin- $\frac{1}{2}$ model on the square lattice, $M_{\mathrm{SUB} 2}^{\mathrm{Z} 2} \approx 0.682$ at $\Delta=$ $\Delta_{c}$. This value is in good agreement with the results of recent large-scale Monte Carlo simulations and the other best available calculations cited in Ref. [14].

We now turn our attention to an alternative approximation scheme which is specifically and closely tailored to lattice systems. The capability of predicting phase transitions in the SUB2 and higher SUB $n$ schemes is clearly related to their inclusion of at least some correlations of arbitrarily long range. However, if we wish to calculate only the energy or other properties of the ground state which are not sensitive to possibly nearby phase transitions, we can employ a very efficient alternative scheme which in practice is capable of very high accuracy. This scheme, denoted as the LSUB $n$ scheme, takes advantage of the localized nature of the interactions, so that one retains at a given order of the approximation only those local configurations in the cluster correlation operators $S$ and $\tilde{S}$ specified by the index $n$. Very specifically, for the problem at hand the LSUB $n$ approximation retains only those configurations 
which contain any number of flipped spins with respect to the Néel model state over a localized region of at most $n$ contiguous sites on the lattice, and which are compatible with the condition that $s_{\text {total }}^{z}=0$.

As a simple example we consider the spin- $\frac{1}{2} 1 \mathrm{D}$ chain. The LSUB2 scheme retains for the operator $S$ only the single coefficient $b_{1} \equiv \mathfrak{S}_{i, i+1}$, whose determining equation from Eq. (30) is given by,

$$
1-2 \Delta b_{1}-3 b_{1}^{2}=0,
$$

with ( physical) solution $b_{1}=\left(\sqrt{\Delta^{2}+3}-\Delta\right) / 3$, and corresponding estimate for the ground-state energy given from Eq. ( 27$)$ as $E_{0} / N=-5 / 12 \approx-0.4167$ for the isotropic $(\Delta=1)$ Heisenberg case. This is surprisingly close to the full SUB2 result of -0.4186 for this case. For the higher-order LSUB $n$ approximations, the number of independent configurations retained in the correlation operator $S$ or $\tilde{S}$ grows rapidly with $n$ (e.g., for $n=4,6,8$, and 10 , the corresponding numbers of independent configurations are $3,9,26$, and 81 , respectively). These are illustrated in Figure 1 for the LSUB6 case. As can be seen from Table I, we obtain ground-state energies with very high accuracy in this LSUB $n$ scheme, even for relatively low values of the truncation index $n$, and even for values of $\Delta<1$ for which the exact ground state has no vestige at all of the perfect long-range order present in the Néel model state.

Turning to the $2 \mathrm{D}$ lattice, it is clear that the number of configurations at a given LSUB $n$ level is considerably higher than in 1D. For example, in LSUB4 approximation for the square lattice there are seven independent spin-flip configurations. However, we can introduce two further parameters in an attempt to categorize the relative importance of the configurations more finely. The first of these is the number $w$ of "wrong" bonds produced by flipping spins with respect to the Néel state. Classically, the breaking of each Néel "up-down" bond in the antiferromagnetic regime costs energy, and hence configurations with the smallest values of $w$ are, a priori, likely to be the most important. Secondly, an extension of the seemingly important concept in 1D of locale size leads us to consider the length $d$ of the "domain boundary" of a given configuration, defined as the number of lattice bonds crossed by a minimalarea circuit (as indicated by the dashed lines in Fig. 2) enclosing all of the flipped spins.

We may thus introduce an LSUB $(w, d)$ approximation for the 2D lattice in which only those spin-flip configurations with values of these parameters not exceeding
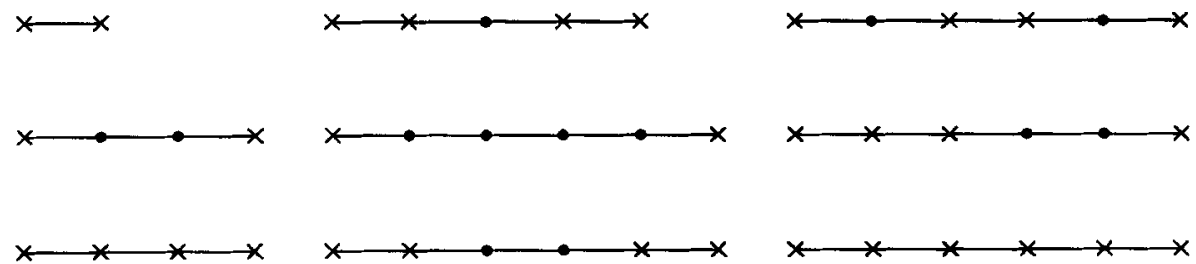

Figure 1. The nine independent configurations of the LSUB6 scheme for the 1D spin- $\frac{1}{2}$ $X X Z$ model. The crosses indicate the flipped spins with respect to the Neel model state. 


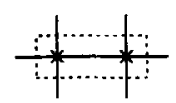

$(6,6)$

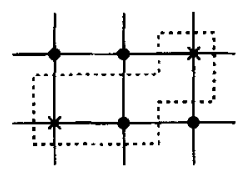

$(8,10)$

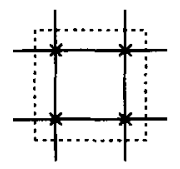

$(8,8)$

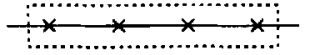

$(10,10)$

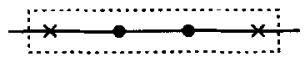

$(8,10)$

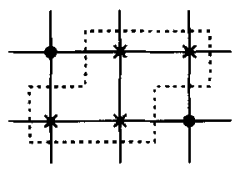

$(10,10)$

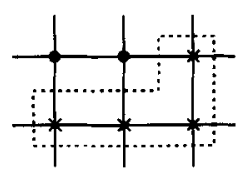

$(10,10)$

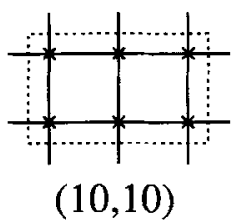

Figure 2. The eight independent configurations of the $\operatorname{LSUB}(10,10)$ scheme for the $2 \mathrm{D}$ spin- $\frac{1}{2} X X Z$ model on a square lattice. The weights $(w, d)$ defined in the text are shown below each configuration.

$w$ and $d$, respectively, are retained. For example, the LSUB $(10,10)$ approximation retains the 8 configurations shown in Figure 2. These include all 7 of the 2D LSUB4 configurations, plus the most compact 6-spin-flip LSUB6 cluster. Results for the ground-state energy of the 2D spin- $\frac{1}{2}$ Heisenberg $(\Delta=1)$ square lattice are shown in Table II. We observe again the excellent convergence properties of our approximation scheme, by comparing with the available Green's function Monte Carlo (GFMC) results [28], and note the marked improvement over the spin-wave theory (SWT) results [29]. It is interesting to note that just as in the LSUBn scheme for the 1D model, our $\operatorname{LSUB}(w, d)$ scheme consistently gives good results for the groundstate energy, for all values of $\Delta \gtrsim 0$. We also point out that our local CCM approximation schemes, such as the LSUB $n$ and $\operatorname{LSUB}(w, d)$ schemes, systematically reproduce the various terms in the perturbation theory series in the $\Delta \rightarrow \infty$ limit [14], at the same time as they provide an effective continuation of the perturbative results far into the nonperturbative regime. This same characteristic of the CCM schemes will be emphasized again in the following applications to lattice gauge theory. Finally, at a rather more pragmatic level, it is also gratifying to note that the $\operatorname{LSUB}(w, d)$ scheme provides a rather effective practical selection of the clusters of greatest significance, to the extent that at any given order the numerical magnitudes of the coefficients of the neglected clusters are, by and large, small in comparison to those retained, for all values of $\Delta \gtrsim 0$. We have verified this observation both by comparison with the $\Delta=1$ results of Ref. [17] and by several calculations of our own carried out to higher orders. 


\section{Applications to Lattice Gauge Field Theory}

We turn now to analogous applications of CCM techniques to lattice gauge theory, where as a specific example we shall consider the Abelian $U(1)$ model of quantum electrodynamics in two discretized spatial plus one continuous time $(2+1)$ dimensions. The $U(1)$ group element on a link $l$ originating at a lattice site with position vector $\mathbf{n}$ in (positive) direction $k$ is $U_{k}(\mathbf{n}) \equiv \exp \left[i A_{k}(\mathbf{n})\right]$, and the lattice Hamiltonian is

$$
H=\frac{1}{2} \sum_{k, \mathbf{n}} E_{k}^{2}(\mathbf{n})+\lambda \sum_{k, \mathbf{n}}\left[1-\cos B_{k}(\mathbf{n})\right],
$$

where $E_{k}(\mathbf{n})$ is the electric field on the links of the lattice and $B_{k}(\mathbf{n})$ is the magnetic field defined as the lattice curl, $B_{i}(\mathbf{n})=\varepsilon_{i j k}\left[A_{k}\left(\mathbf{n}+\mathbf{e}_{j}\right)-A_{k}(\mathbf{n})\right]$ around the elementary square plaquettes defined by the unit lattice vectors $\mathbf{e}_{i}$. Quantum mechanics is imposed in the temporal gauge through the basic commutation relation, $\left[A_{k}(\mathbf{n})\right.$, $\left.E_{k^{\prime}}\left(\mathbf{n}^{\prime}\right)\right]=i \delta_{k k^{\prime}} \delta_{n \mathbf{n}^{\prime}}$, which is realizable via the representation $E_{k}(\mathbf{n}) \rightarrow-i \partial / \partial A_{k}(\mathbf{n})$. The Hamiltonian may then be represented wholly in terms of the plaquette variables, $B_{p}\left(\equiv B_{k}(\mathbf{n})\right)$, in the gauge-invariant sector. For example, in $2+1$ dimensions, we find

$$
H=\sum_{p}\left[-2 \frac{\partial^{2}}{\partial B_{p}^{2}}+\lambda\left(1-\cos B_{p}\right)\right]+\sum_{\left\langle p, p^{\prime}\right\rangle} \frac{\partial^{2}}{\partial B_{p} \partial B_{p^{\prime}}},
$$

where the second sum over $\left\langle p, p^{\prime}\right\rangle$ indicates all nearest neighbor pairs of plaquettes. Equation (36) thus defines an infinite many-body problem with compact variables, $-\pi<B_{p} \leq \pi$.

In the non-Abelian $S U(N)$ case the basic variables are the $S U(N)$ matrices defined on each link $l$ in terms of the $N^{2}-1$ group generators, and the conjugate "chromoelectric" fields $E_{l}^{\alpha}$ have $N^{2}-1$ components. Thus, for $S U(2)$ the group element on each link has the general form $U=d_{0} \mathbf{l}+i \mathbf{d} \cdot \sigma$, where the $\sigma_{k}$ are the usual Pauli matrices, and the real coefficients $d_{\alpha}$ lie on a sphere in four-dimensional Euclidean space, $d_{0}^{2}+\sum_{k=1}^{3} d_{k}^{2}=1$. The potential term in Eq. (36) generalizes to $\lambda \sum_{p}(N-$ $\operatorname{Re} \operatorname{Tr} U_{p}$ ), where $U_{p} \equiv U_{1} U_{2} U_{3}^{\dagger} U_{4}^{\dagger}$ for the plaquette formed from the four links $l=$ $1,2,3,4$ in cyclic order.

Our main goal is now to provide a CCM parametrization of the physical (i.e., gauge-invariant) ground state and excited ("glueball") states of the above Hamiltonian in the vacuum sector $[22,24]$. We thus need firstly to choose a model state $|\Phi\rangle$. A convenient choice is the so-called electric $(\lambda \rightarrow 0)$ vacuum $|0\rangle$, for which $E_{l}|0\rangle=0$ for all $l$. The CCM now parametrizes the exact ground state in terms of $|\Phi\rangle \rightarrow|0\rangle$ as in Eq. (4), with a cluster correlation operator $S$ given by a sum over all possible linearly independent linked clusters of Wilson loops,

$$
S=\sum_{\Gamma} \sum_{p} \Re_{\Gamma} A_{\Gamma}(p),
$$

with $\mathrm{T}\left(\equiv\left\{\gamma_{i} ; i=1,2, \cdots, n\right\} ; n=1,2, \cdots\right)$ and $p$ denoting the geometry and position of the oriented loop clusters respectively, and $A_{\Gamma}=A_{\gamma_{1}} \cdots A_{\gamma_{n}}$, with 
$A_{\gamma} \equiv \operatorname{Tr}[U \cdots U]_{\gamma}$ for each closed loop $\gamma$. The ground-state energy $E_{0}$ and correlation coefficients $\left\{\mathfrak{e}_{\Gamma}\right\}$ are determined as in Eqs. (7) and (8), respectively, with $C_{J} \rightarrow A \ddagger(p)$, and where the inner products incorporate the appropriate group measure. Furthermore, for the Hamiltonian of Eq. (36) and the correlation operator of Eq. (37), the expansion of Eq. (9) terminates at the term of second order in $S$.

Henceforth we focus on the specific $U(1)$ model of Eq. (36) in $2+1$ dimensions. (In earlier work [24] we have studied the same $U(1)$ model in $1+1$ dimensions, which has similar qualitative behavior. By contrast, the same model in $3+1 \mathrm{di}$ mensions is expected to show marked differences.) The electric vacuum state is thus simply a constant. Furthermore, the operator $S$ can be partitioned into $k$ plaquette pieces, $S=\sum_{k=1}^{N_{\underline{p}}} S_{k}$, where $N_{p} \rightarrow \infty$ is the total number of elementary square plaquettes in the lattice. We have, for example,

$$
\begin{gathered}
S_{1}=\sum_{n=1}^{\infty} \sum_{p=1}^{N_{p}} \mathcal{S}_{p}(n) \cos n B_{p} ; \\
S_{2}=\sum_{n, n^{\prime}=1}^{\infty} \sum_{p, p^{\prime}=1}^{N_{p}}\left[\mathcal{S}_{p, p^{\prime}}^{(1)}\left(n, n^{\prime}\right) \cos n B_{p} \cos n^{\prime} B_{p^{\prime}}\right. \\
\left.\quad+\mathcal{S}_{p, p^{\prime}}^{(2)}\left(n, n^{\prime}\right) \sin n B_{p} \sin n^{\prime} B_{p^{\prime}}\right],
\end{gathered}
$$

where the prime on the sum in Eq. (39) excludes the term with $p=p^{\prime}$. For this case we have $S^{\dagger}=S$, and the inner products in the coupled equations of Eqs. (7) and $(8)$ are simply the multiple integrals over all plaquette variables $\left\{B_{p}\right\}$ within the range $-\pi<B_{p}<\pi$. We also note that the multi-plaquette correlation operators $\left\{S_{k}\right\}$ have a close relation to the usual Wilson loops $A_{\Gamma}$ mentioned above. For example, the simple trigonometric relation, $2 \cos B_{1} \cos B_{2}=\cos \left(B_{1}+B_{2}\right)+$ $\cos \left(B_{1}-B_{2}\right)$ corresponds to the relationship between Wilson loops illustrated in Figure 3. Our particular parametrization exemplified by Eqs. (38) and (39) is not only complete but is also especially useful in view of the orthonormality of the basis.

It should now be immediately clear that the multi-plaquette correlations in the exact ground state $\left|\Psi_{0}\right\rangle$ can be approximated by truncating the operator $S$ using the analogues of the schemes described previously for the spin-lattice systems. Further subtruncations are also possible here, in terms of the numbers of modes $\left\{n_{i}\right\}$ kept in the summations in Eqs. (38) and (39) and their higher-order counterparts. For example, the LSUB $n(m)$ scheme ignores, firstly, all terms in $S$ between $k>n$ plaquettes and between $k<n$ plaquettes if these $k$ plaquettes occupy a region which cannot be delimited by at most $n$ contiguous plaquettes on the lattice; and, secondly, all terms in the resulting LSUB $n$ correlation operator with $\Sigma_{i} n_{i}>m$. The mode

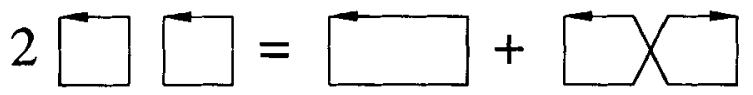

Figure 3. Graphical illustration of a relationship between the $U(1)$ Wilson loops. 
numbers $\left\{n_{l}\right\}$ clearly bear a direct relationship with the winding numbers of the equivalent Wilson loops retained in the sum for $S$. Thus, it should also be clear how to generalize our approach to the non-Abelian $S U(N)$ lattice gauge theories.

The ground-state energy for our $U(1)$ model in $2+1$ dimensions is shown in Figure 4 and Table III as a function of $\lambda$, as obtained in the SUB1 and LSUB2 $(m)$ schemes. The full SUB 1 calculation is completely equivalent to the one-body Mathieu problem, and the SUB $1(\mathrm{~m})$ subtruncations are found to converge extremely rapidly with increasing $m$ even for large values of $\lambda$. We also include for comparison some results of $n$ th-order strong-coupling $(\lambda \rightarrow 0)$ perturbation theory (denoted as $\operatorname{PT} n(\mathrm{~S}))$; lowest-order weak-coupling $(\lambda \rightarrow \infty)$ perturbation theory $(\operatorname{PT}(W))$; the results from an analytic continuation of the strong-coupling perturbation series due to Hamer, Oitmaa, and Zheng ( $\mathrm{HOZ}$ ) [30]; and those from the $t$-expansion calculation of Morningstar [31]. One sees that our LSUB2 $(m)$ results also converge quickly as $m$ increases; and that the LSUB2(10) results are in very good agreement at this quite low order of approximation with those from the careful analysis of Morningstar, even for large values of $\lambda$ well into the weak-coupling regime.

\section{Discussion}

It is interesting to note that for the $X X Z$ spin-lattice model discussed, the LSUBn approximation exactly reproduces the results of $n$ th-order perturbation theory in

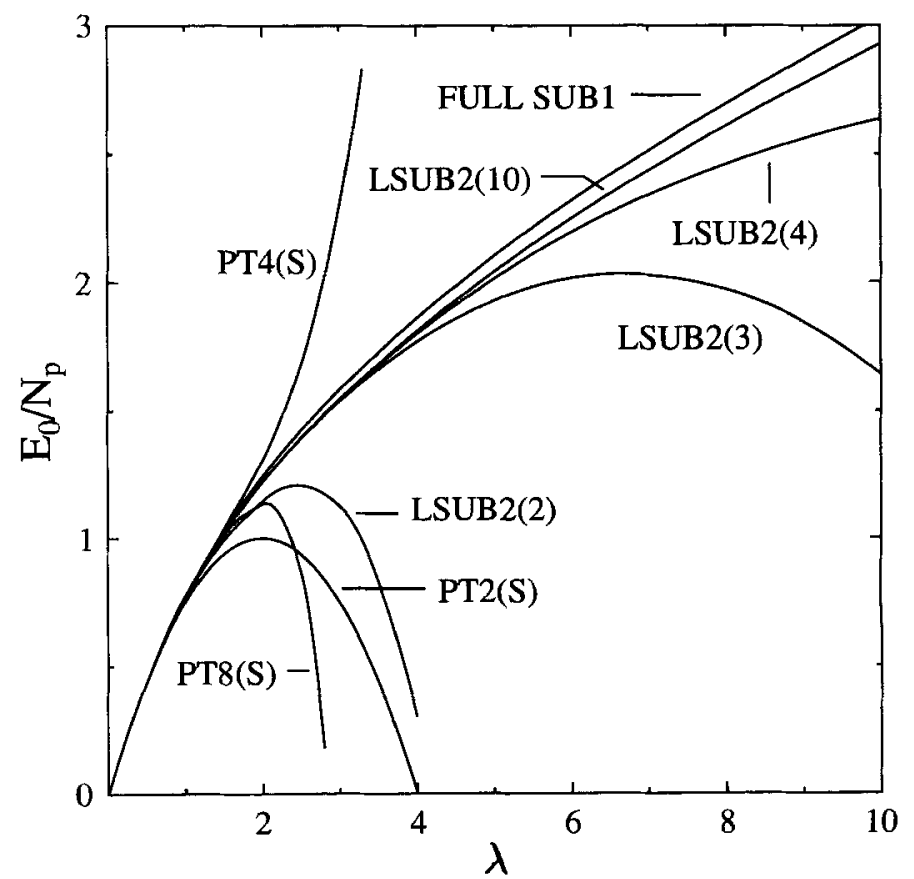

Figure 4. Ground-state energy per plaquette of the $U(1)$ model on the square lattice. 
TABLE III. Ground-state energy per plaquette at several values of $\lambda$ for the $U(1)$ model in $2+1$ dimensions. Shown are the results of various LSUB2 $(n)$ schemes, together with the full sub1 results and the results of both strong $(\lambda \rightarrow 0)$ and weak $(\lambda \rightarrow \infty)$ coupling limits in perturbation theory. We show the results of 4th-and 8th-order strong-coupling perturbation theory, PT4(S) and PT8(S) respectively, and of lowest-order weak-coupling perturbation theory, $\mathrm{PT}(\mathrm{W})$. The results from an analytic continuation of the strong-coupling perturbation series by Hamer, Oitmaa, and Zheng [30] (denoted as HOZ), and

from the $t$-expansion calculations of Morningstar [31], are also included for comparison purposes.

\begin{tabular}{|c|c|c|c|c|c|c|c|c|c|}
\hline \multirow[b]{2}{*}{ Method } & \multicolumn{9}{|c|}{$\lambda$} \\
\hline & 0.5 & 1 & 2 & 3 & 4 & 5 & 6 & 8 & 9 \\
\hline SUB 1 & 0.4391 & 0.7724 & 1.2430 & 1.5828 & 1.8597 & 2.1000 & 2.3156 & 2.6966 & 2.8688 \\
\hline LSUB $2(2)$ & 0.4386 & 0.7652 & 1.1468 & 1.1280 & 0.3019 & -2.8326 & -15.108 & - & - \\
\hline LSUB $2(3)$ & 0.4387 & 0.7681 & 1.2216 & 1.5371 & 1.7691 & 1.9282 & 2.0153 & 1.9720 & 1.8424 \\
\hline LSUB2(4) & 0.4387 & 0.7681 & 1.2214 & 1.5428 & 1.7994 & 2.0123 & 2.1921 & 2.4585 & 2.5568 \\
\hline LSUB $2(6)$ & 0.4387 & 0.7681 & 1.2217 & 1.5453 & 1.8099 & 2.0404 & 2.2482 & 2.6188 & 2.7886 \\
\hline LSUB2(8) & 0.4387 & 0.7681 & 1.2217 & 1.5454 & 1.8100 & 2.0404 & 2.2477 & 2.6142 & 2.7797 \\
\hline LSUB2(10) & 0.4387 & 0.7681 & 1.2216 & 1.5452 & 1.8095 & 2.0393 & 2.2456 & 2.6096 & 2.7734 \\
\hline $\mathrm{HOZ}$ & - & - & 1.215 & - & 1.785 & - & 2.2 & - & - \\
\hline PI 4(S) & 0.4387 & 0.7690 & 1.3042 & 2.2898 & 4.8667 & 10.632 & 21.638 & - & - \\
\hline PT8(S) & 0.4387 & 0.7673 & 1.1358 & -0.7375 & -20.873 & - & - & - & - \\
\hline PT(W) & 0.5627 & 0.8434 & 1.2402 & 1.5447 & 1.8015 & 2.0276 & 2.2321 & 2.5917 & 2.7596 \\
\hline Morningstar & - & 0.7675 & - & - & 1.796 & - & - & - & 2.763 \\
\hline
\end{tabular}

the limit $\Delta \rightarrow \infty[14]$. The strong-coupling $(\lambda \rightarrow 0)$ perturbation series is similarly reproduced from our LSUB $n$ equations for the $U(1)$ lattice gauge theory [22,24]. Thus, our LSUB $n$ approximations effectively comprise a well-defined analytic continuation or resummation of such perturbation series within the context of a rather natural and consistent hierarchical scheme. From the numerical results obtained, the CCM approach is seen to be far superior in this sense to the alternative rather ad hoc approaches for extending the range of validity or the accuracy of similar perturbative sequences, which are usually based on generalized Padé approximants or similar techniques.

From the results obtained to date we hope to have convinced the reader that the CCM approach indeed provides a unified many-body theory of quantal Hamiltonian lattice systems. Obvious extensions then present themselves as worthy of study. For the spin-lattice systems it is of interest to investigate other models, such as the spin1 Heisenberg-biquadratic chain [16], or spin- $\frac{1}{2}$ chains with both nearest-neighbor and next-nearest-neighbor couplings. In this context the use of alternative model states $|\Phi\rangle$ is of particular interest. For the lattice gauge models we are also interested in extending our very preliminary work done so far in the vacuum sector on the $U$ (1) excitation gap (or glueball mass) [24] and on the non-Abelian $S U(2)$ model. In principle, the work described above for the vacuum sector can also be extended to the charged (or "colored") sector by the further inclusion in the correlation operator $S$ of sums over open paths on the lattice, representing tubes of electric flux between staggered fermions, as well as the sums over closed paths (i.e., the Wilson loops) already considered. 
Finally, the quality of our results obtained so far convinces us that the extension of the current CCM approach to additional Hamiltonian lattice systems is well justified. An obvious example is the class of strongly interacting electronic lattice systems, including such topical models as the Hubbard and $t J$ models which, despite intensive recent study by various theoretical techniques, are still only partially understood. Preliminary work in this direction has been reported [19-21], and we hope to present results of our own soon.

\section{Acknowledgments}

We thank our collaborators J. B. Parkinson and R. G. Hale in the case of the spin-lattice models, and A. S. Kendall and L. Y. Wong in the case of lattice gauge theory, for their assistance. One of us (RFB) also gratefully acknowledges the support of a research grant from the Science and Engineering Research Council (SERC) of Great Britain.

\section{Bibliography}

[1] R. J. Bartlett, J. Phys. Chem. 93, 1697 (1989).

[2] R. J. Bartlett, Theor. Chim. Acta 80, 71 ( 1991 ) -and note also that issues 2-6 of this volume are entirely devoted to articles on the coupled cluster theory of electron correlations in many-electron systems.

[3] R. F. Bishop, Theor. Chim. Acta 80, 95 (1991).

[4] R. F. Bishop and K. H. Lührmann, Phys. Rev. B 17, 3757 (1978).

[5] J. Arponen, Ann. Phys. (NY) 151, 311 (1983).

[6] H. Kümmel, in Nucleon-Nucleon Interaction and Nuclear Many-Body Problems, S. S. Wu and T. T. S. Kuo, Eds. (World Scientific, Singapore, 1984), p. 46.

[7] R. F. Bishop and H. G. Kümmel, Phys. Today 40(3), 52 (1987).

[8] J. S. Arponen, R. F. Bishop, and E. Pajanne, Phys. Rev. A 36, 2519 (1987); 36, 2539 (1987).

[9] R. F. Bishop, Int. J. Quantum Chem., Quantum Chem. Symp. 24, 197 (1990).

[10] J. S. Arponen and R. F. Bishop, Ann. Phys. (NY) 207, 171 (1991).

[11] R. F. Bishop, in Dirkfest '92: A Symposium in Honor of J. Dirk Walecka's Sixtieth Birthday, W. W. Buck, K. M. Maung, and B. D. Serot, Eds. (World Scientific, Singapore, 1992), p. 21.

[12] J. S. Arponen and R. F. Bishop, Ann. Phys. (NY) 227, 275 (1993); 227, 334 (1993).

[13] M. Roger and J. H. Hetherington, Phys. Rev. B 41, 200 (1990).

[14] R. F. Bishop, J. B. Parkinson, and Yang Xian, Phys. Rev. B 43, 13782 ( 1991 ); 44, 9425 (1991); Theor. Chim. Acta 80, 181 (1991); in Condensed Matter Theories, Vol. 6, S. Fantoni and S. Rosati, Eds. (Plenum, New York, 1991), p. 37; J. Phys.: Condens. Matter 4, 5783 (1992); in Recent Progress in Many-Body Theories, Vol. 3, T. L. Ainsworth, C. E. Campbell, B. E. Clements, and E. Krotscheck, Eds. (Plenum, New York, 1992), p. 117.

[15] R. F. Bishop, J. B. Parkinson, and Yang Xian, Phys. Rev. B 46, 880 (1992).

[16] R. F. Bishop, J. B. Parkinson, and Yang Xian, J. Phys.: Condens. Matter 5, 9169 (1993).

[17] F. E. Harris, Phys. Rev. B 47, 7903 (1993).

[18] F. Cornu, Th. Jolicouer, and J. C. Le Guillou, CEA-Saclay preprint T93/120 (1993).

[19] M. Roger and J. H. Hetherington, Europhys. Lett. 11, 255 (1990).

[20] C. F. Lo, E. Manousakis, and Y. L. Wang, Phys. Lett. A156, 42 (1991).

[21] F. Petit and M. Roger, Phys. Rev. B (1994), in press.

[22] R. F. Bishop and Yang Xian, Acta Phys. Polonica B 24, 541 (1993).

[23] C. H. Llewellyn Smith and N. J. Watson, Phys. Lett. B 302, 463 (1993).

[24] R. F. Bishop, A. S. Kendall, L. Y. Wong, and Yang Xian, Phys. Rev. D 48, 887 (1993); in Condensed Matter Theories, Vol. 8, L. Blum and F. B. Malik, Eds. (Plenum, New York, 1993), p. 269. 
[25] H. A. Bethe, Z. Phys. 71, 205 (1931); L. Hulthén, Ark. Mat. Astron. Fys. A26, No. 11 (1938); J. des Cloiseaux and J. J. Pearson, Phys. Rev. 128, 2131 (1962); L. D. Faddeev and L. A. Takhtajan, Phys. Lett. A85, 375 (1981).

[26] F. D. M. Haldane, Phys. Lett. A93, 464 (1983); Phys. Rev. Lett. 50, 1153 (1983).

[27] K. Emrich, Nucl. Phys. A351, 379 (1981); A351, 397 ( 1981 ).

[28] N. Trivedi and D. M. Ceperley, Phys. Rev. B 41, 4552 (1990).

[29] P. W. Anderson, Phys. Rev. 86, 694 (1952); T. Oguchi, Phys. Rev. 117, 117 (1960).

[30] C. J. Hamer, J. Oitmaa, and W. H. Zheng, Phys. Rev. D 45, 4652 (1991).

[31] C. J. Morningstar, Phys. Rev. D 46, 824 (1992).

Received March 28, 1994 